\title{
Quantum Time Crystals from Hamiltonians with Long-Range Interactions
}

\author{
Valerii K. Kozin $\oplus^{1,2}$ and Oleksandr Kyriienko $\oplus^{3,4,5}$ \\ ${ }^{1}$ Science Institute, University of Iceland, Dunhagi 3, IS-107 Reykjavik, Iceland \\ ${ }^{2}$ ITMO University, Kronverkskiy prospekt 49, Saint Petersburg 197101, Russia \\ ${ }^{3}$ Department of Physics and Astronomy, University of Exeter, Stocker Road, Exeter EX4 4QL, United Kingdom \\ ${ }^{4}$ Department of Nanophotonics and Metamaterials, ITMO University, St. Petersburg, 197101, Russia \\ ${ }^{5}$ NORDITA, KTH Royal Institute of Technology and Stockholm University, Roslagstullsbacken 23, SE-106 91 Stockholm, Sweden
}

(Received 23 July 2019; published 20 November 2019)

\begin{abstract}
Time crystals correspond to a phase of matter where time-translational symmetry (TTS) is broken. Up to date, they are well studied in open quantum systems, where an external drive allows us to break discrete TTS, ultimately leading to Floquet time crystals. At the same time, genuine time crystals for closed quantum systems are believed to be impossible. In this study we propose a form of a Hamiltonian for which the unitary dynamics exhibits the time crystalline behavior and breaks continuous TTS. This is based on a spin-1/2 many-body Hamiltonian which has long-range multispin interactions in the form of spin strings, thus bypassing previously known no-go theorems. We show that quantum time crystals are stable to local perturbations at zero temperature. Finally, we reveal the intrinsic connection between continuous and discrete TTS, thus linking the two realms.
\end{abstract}

DOI: 10.1103/PhysRevLett.123.210602

Introduction.-Crystalline structures are ubiquitous in nature, and surround us in everyday life. Formally, a crystal is defined as a structure of atoms that is periodically ordered in space, and is stable to external perturbations [1]. Generalized to various other constituents, the concept was found applicable in many areas of science, including photonic crystals in optics [2,3], synthetic cold atom lattices [4], granular materials [5], etc. While thought to be inherent to the spacial degrees of freedom, the contemporary understanding of physics in space-time posed the question of the possibility of having a crystalline structure in the time dimension. This would correspond to a timeperiodic behavior, which persists perpetually. For the classical case, the time crystal (TC) was described by Shapere and Wilczek [6]. Finally, the quantum case was postulated by Frank Wilczek [7], theorizing the breaking of time-translational symmetry. This was suggested to be observed in superconducting rings [8] or trapped ion [9] systems. However, despite the fact that the basic time crystal formulation remains an intriguing concept, the subsequent studies have shown that ground state TC cannot be realized in the simple setting [10,11], and are ruled out by the no-go theorem.

The formalized definition of a quantum time crystal was set by Watanabe and Oshikawa (W-O) [12], who proposed to closely follow the definition for spatial crystals. It should be noted, however, that there is a difference between breaking the space and time-translational symmetries. In the case of spontaneous breaking of the space translational symmetry the ground state is highly degenerate in the thermodynamic limit. This results in the fact that the ground state of a crystal does not have a definite momentum even though the Hamiltonian commutes with the momentum operator. The continuous time translational symmetry is present in isolated systems where the Hamiltonian does not depend on time (the Hamiltonian commutes with the evolution operator) but the ground state must have a certain energy by definition. Instead, W-O defined the condition for the correlation function to be a periodic function of time $t$ as

$$
\lim _{N \rightarrow \infty}\langle\hat{\Phi}(t) \hat{\Phi}(0)\rangle=f(t)
$$

where the operator $\hat{\Phi}(t)$ corresponds to an integrated order parameter (e.g., average density or magnetization) at time $t$, and the averaging over the ground state of the system is considered in the thermodynamic limit of an infinite number of lattice sites $N$. At the same time, W-O have proven that nonvanishing periodic oscillations are impossible unless two conditions are violated: (i) the system is out-of-equilibrium; or (ii) the interaction is of the longrange type, decaying slower than power law $\sim r^{-\alpha}$ for $\alpha>0$. This left the question of possibility for quantum time crystals to remain in the air.

Analyzing the first constraint, the consideration of the driven quantum system previously has allowed breaking TTS in discrete time [13]. The idea relies on the successive application of driver and interaction unitaries, and ultimately led to the appearance of Floquet or discrete time crystals (DTC) [13-15]. For the chain of spin- $1 / 2$ particles, the protocol reads as simultaneous spin flip application to 
the entire chain, which leads to the oscillations of magnetization, followed by Ising type interaction unitary. The major feature of the described system is a subharmonic response which is stable to drive perturbations $[14,16]$. As the driven system is prone to heating in the high frequency limit, it also involves many-body localized unitary evolution, although later this condition was relaxed if the finite timescale is considered [17-19]. The successful theoretical prediction of time-periodic structures in driven systems was followed by its experimental observation in trapped ions [20], silicon vacancy-based quantum simulators [21], superfluid helium-3 [22], and NMR systems [23,24]. Finally, many works targeting other platforms and generalizations were presented, including $\mathrm{TC}$ in fermionic lattices [18] and Majorana fermions [25], dissipative DTCs [26], topological DTCs [27], discrete time quasicrystals [28], cosmological space-time crystals [29,30], and described the dynamical phase transition for DTCs [31]. The emergent field up to date was reviewed in several indepth studies [32,33]. Further directions represent exploration of quantum scar physics in nonequilibrium setting [3437] or modification of $\mathrm{TC}$ definition to nonlocal order parameters [38]. Finally, to study the breaking of continuous TTS, several open system realizations were considered up to date, including fermionic cold atom lattices [39] and Rydberg atoms [40].

Although the number of systems demonstrating discrete time crystalline behavior grows rapidly for driven-dissipative and Floquet systems, the original issue of possibility to break continuous time-translational symmetry and existence of genuine time crystals for a closed quantum system remains an open challenge. In this Letter we use the original time crystal definition and exploit the second loophole in the W-O no-go theorem to show that there is a class of static Hamiltonians which host the TC phase for the unitary dynamics. In particular, using the W-O definition for a spin-1/2 many-body system, we find a Hamiltonian with spin string-type interactions which corresponds to a system with a ground state being a nondegenerate maximally entangled state. The minimal size of the spin string to support the TC phase is $N / 2$ for a system of $N$ particles, thus formally bypassing the applicability range of the W-O theorem [12]. The stability of the system is studied, showing resilience to local perturbations.

Continuous time crystal Hamiltonian.-We start by postulating that certain many-body Hamiltonians that contain terms involving all particles can support the time crystalline phase and exhibits spontaneously broken continuous TTS. Here, we present a method of constructing a Hamiltonian satisfying the W-O definition of a quantum time crystal. To be concrete, we focus on an $N$ qubit system, and as an operator $\hat{\Phi}$ in the definition (1) we consider the total magnetization $\hat{M}_{z}=\sum_{i=1}^{N} \sigma_{z}^{(i)} / N$ along the $z$ axis, where $\sigma_{x, y, z}^{(i)}$ denote Pauli matrices acting on qubits at site $i$. In this case, the Watanabe-Oshikawa TC definition in Eq. (1) recasts as $\lim _{N \rightarrow \infty}\left\langle\hat{M}_{z}(t) \hat{M}_{z}(0)\right\rangle=$ $f(t)$, where $f(t)$ must be periodic. To construct a Hamiltonian satisfying this definition we start with a state $\left|E_{0}\right\rangle$ from the Hilbert space that we chose as a ground state at energy $\epsilon_{0}$. Acting on $\left|E_{0}\right\rangle$ by a nonunitary operator $\hat{M}_{z}$ and renormalizing the vector gives the partner state $\left|E_{1}\right\rangle=\hat{M}_{z}\left|E_{0}\right\rangle / \sqrt{\left\langle E_{0}\left|\hat{M}_{z}^{2}\right| E_{0}\right\rangle}$. The crucial point here is to demand that $\left|E_{0}\right\rangle$ is chosen such that $\left|E_{1}\right\rangle$ is orthogonal to $\left|E_{0}\right\rangle$, i.e., $\left\langle E_{0}\left|\hat{M}_{z}\right| E_{0}\right\rangle=0$. Having done that, we assign an energy $\epsilon_{1}>\epsilon_{0}$ to the state $\left|E_{1}\right\rangle$, and construct the Hamiltonian using projectors for the many-body states as

$$
\hat{H}=\epsilon_{0}\left|E_{0}\right\rangle\left\langle E_{0}\left|+\epsilon_{1}\right| E_{1}\right\rangle\left\langle E_{1}\left|+\sum_{j>1} \epsilon_{j}\right| E_{j}\right\rangle\left\langle E_{j}\right|,
$$

where arbitrary real numbers $\epsilon_{1}, \epsilon_{2}, \ldots, \epsilon_{2^{N}-1}$ must all be greater than $\epsilon_{0}$, with $\epsilon_{0}$ being a ground state energy, and arbitrary state vectors are chosen such that $\left\langle E_{0,1} \mid E_{j}\right\rangle=0$ for $j>1$. We claim that Eq. (2) describes the quantum time crystal Hamiltonian if certain restrictions on $\left|E_{0}\right\rangle$ are respected. To find these restrictions we calculate the correlation function at finite size $N$ of the system given in Eq. (1) for the Hamiltonian (2), which yields

$$
\begin{aligned}
f_{N}(t) & =\left\langle E_{0}\left|\hat{M}_{z}(t) \hat{M}_{z}(0)\right| E_{0}\right\rangle=\left\langle E_{0}\left|e^{i \hat{H} t} \hat{M}_{z} e^{-i \hat{H} t} \hat{M}_{z}\right| E_{0}\right\rangle \\
& =e^{i \epsilon_{0} t}\left\langle E_{0}\left|\hat{M}_{z}^{2}\right| E_{0}\right\rangle\left\langle E_{1}\left|e^{-i \hat{H} t}\right| E_{1}\right\rangle=\mathcal{O} e^{-i\left(\epsilon_{1}-\epsilon_{0}\right) t},
\end{aligned}
$$

where $\mathcal{O}=\left\langle E_{0}\left|\hat{M}_{z}^{2}\right| E_{0}\right\rangle$ denotes the order parameter. If it remains nonzero $\mathcal{O} \neq 0$ at $N \rightarrow \infty$ (as for the case of longrange order in crystals [12]) and the energy difference $\omega=\epsilon_{1}-\epsilon_{0} \neq 0$, then $\lim _{N \rightarrow \infty} f_{N}(t)=f(t)$ is a periodic function of time in the thermodynamic limit, oscillating at frequency $\omega$. The latter condition on $\omega$ can be fulfilled easily by construction, whereas the former one needs an additional consideration.

We remind that our goal is to find such a state $\left|E_{0}\right\rangle$ that (a) $\left\langle E_{0}\left|\hat{M}_{z}\right| E_{0}\right\rangle=0$ for any $N$, and (b) $\mathcal{O}$ remains nonzero at $N \rightarrow \infty$. In order to analyze the conditions (a) and (b) it is convenient to expand $\left|E_{0}\right\rangle$ in the eigenbasis of the Hermitian operator $\hat{M}_{z}$. The operator $\hat{M}_{z}$ has $N+1$ distinct eigenvalues $m_{k}=(N-2 k) / N$, where $k=0,1, \ldots, N$ each having degeneracy $C_{N}^{k}$. Denoting the corresponding eigenstates as $\left|m_{k}, d\right\rangle$, where $d=1,2, \ldots, C_{N}^{k}$ is accounting for degeneracy, we can express $\left|E_{0}\right\rangle=\sum_{k, d} c_{k, d}\left|m_{k}, d\right\rangle$, where $c_{k, d}$ are normalized to unity, giving $\left\langle E_{0}\left|\hat{M}_{z}\right| E_{0}\right\rangle=$ $\sum_{k, d}\left|c_{k, d}\right|^{2} m_{k} \quad$ and $\quad\left\langle E_{0}\left|\hat{M}_{z}^{2}\right| E_{0}\right\rangle=\sum_{k}\left|c_{k, d}\right|^{2} m_{k}^{2}$. The eigenbasis $\left|m_{k}, d\right\rangle$ corresponds to states having $k$ spins pointing down and $N-k$ pointing up, and vice versa if we replace $k$ with $N-k\left(m_{k}=-m_{N-k}\right)$. Thus, in order to fulfill condition (a) we can choose any superposition such that $\left|c_{k, d}\right|=\left|c_{N-k, d}\right|$.

One of the examples of the states satisfying the requirements discussed above, is the pair of maximally entangled Greenbergen-Horne-Zeilinger (GHZ) states [41] 


$$
\left|G_{\mp}\right\rangle=\frac{1}{\sqrt{2}}(|\uparrow \uparrow \ldots \uparrow\rangle \mp|\downarrow \downarrow \ldots \downarrow\rangle),
$$

as they fulfill $\hat{M}_{z}\left|G_{-,+}\right\rangle=\left|G_{+,-}\right\rangle$and $\left\langle G_{-,+} \mid G_{+,-}\right\rangle=0$. Thus, the simplest possible TC Hamiltonian can be constructed as

$$
\hat{H}=-\left|G_{+}\right\rangle\left\langle G_{+}\right|,
$$

where, in accordance with the notation introduced earlier, $\epsilon_{0}=-1, \epsilon_{1}=\epsilon_{2}=\ldots=\epsilon_{2^{N}-1}=0$.

If the ground state is $m$ degenerate, $m$ remains finite at $N \rightarrow \infty$, and for each $i=1, \ldots, m$ ground state $\left|E_{0}^{\{i\}}\right\rangle$ the quantity $\left\langle E_{0}^{\{i\}}\left|\hat{M}_{z}^{2}\right| E_{0}^{\{i\}}\right\rangle$ does not vanish in the thermodynamic limit, then the system remains a quantum time crystal for the averaging in Eq. (1) performed over the zero temperature density matrix

$$
\hat{\rho}=\frac{1}{m} \sum_{i=1}^{m}\left|E_{0}^{\{i\}}\right\rangle\left\langle E_{0}^{\{i\}}\right|
$$

Moreover, the same conclusion holds for nonzero temperature once the contribution of excited states $\left|\left\langle E_{j}\left|\hat{M}_{z}\right| E_{k}\right\rangle\right|^{2}$ remains finite in $N \rightarrow \infty$ limit.

Generalizing the zero temperature case considered above, we proceed to the case of nonzero temperature $T=(k \beta)^{-1}$, when the system is described by the density matrix $\hat{\rho}=Z^{-1} e^{-\beta \hat{H}}$, where $Z=\sum_{i} e^{-\beta \epsilon_{i}}$. Without loss of generality, we focus on the system described by the Hamiltonian (2) having the nondegenerate ground state. Then, the correlation function (1) for finite $N$ reads

$$
\begin{aligned}
f_{N}(t)= & Z^{-1} e^{-\beta \epsilon_{0}}\left\langle E_{0}\left|\hat{M}_{z}^{2}\right| E_{0}\right\rangle e^{i\left(\epsilon_{0}-\epsilon_{1}\right) t} \\
& +Z^{-1} \sum_{j>0} e^{-\beta \epsilon_{j}} e^{i \epsilon_{j} t}\left\langle E_{j}\left|\hat{M}_{z} e^{-i \hat{H} t} \hat{M}_{z}\right| E_{j}\right\rangle,
\end{aligned}
$$

and inserting the unity decomposition in the last line right after $e^{-i \hat{H} t}$ we obtain

$$
\begin{aligned}
f_{N}(t)= & Z^{-1} e^{-\beta \epsilon_{0}}\left\langle E_{0}\left|\hat{M}_{z}^{2}\right| E_{0}\right\rangle e^{i\left(\epsilon_{0}-\epsilon_{1}\right) t} \\
& +Z^{-1} \sum_{j>0} \sum_{k \geq 0} e^{-\beta \epsilon_{j}} e^{i\left(\epsilon_{j}-\epsilon_{k}\right) t}\left|\left\langle E_{j}\left|\hat{M}_{z}\right| E_{k}\right\rangle\right|^{2} .
\end{aligned}
$$

From the analysis of Eq. (8) we conclude that if there is a finite number of non-zero terms $\left\langle E_{j}\left|\hat{M}_{z}\right| E_{k}\right\rangle$ in the thermodynamic limit and $Z$ does not diverge in the same limit, then the system represents a quantum time crystal for any temperature.

Locality of the TC Hamiltonian.-We have presented the quantum time crystal Hamiltonian by construction, formulated in terms of the many-body projector and coupling operators. However, when it is rewritten in terms of physical interaction between qubits [e.g., taking the toy
TC Hamiltonian from Eq. (5)], it reveals highly nonlocal character. One can easily check that the resulting expression contains $N$-length spin strings of $\sigma_{x}$ operators with pairwise substitution of $\sigma_{y}^{(i)} \sigma_{y}^{(j)}$ couples, and even-length strings of $\sigma_{z}$ operators [42]. This corresponds to the sum of GHZ-state stabilizer products [41], which must satisfy the conditions specified for Hamiltonian (2). While there are various ways the spin strings can be combined, one can consider a particular example and verify that W-O conditions for TC are satisfied. For instance, taking the Hamiltonian of an $\mathrm{N}$-qubit system in the form

$\hat{H}=-\frac{J}{N(N-1)} \sum_{1 \leq i<j \leq N} \sigma_{x}^{(1)} \sigma_{x}^{(2)} \ldots \sigma_{y}^{(i)} \ldots \sigma_{y}^{(j)} \ldots \sigma_{x}^{(N)}$,

where there are exactly two $\sigma_{y}$ operators in each term filled with $\sigma_{x}$ 's, we observe that its spectrum is bounded between $-J / 2 \leq \epsilon_{n} \leq J / 2$. The nondegenerate ground state of the Hamiltonian (9) is given by $\left|G_{-}\right\rangle$with energy $\epsilon_{0}=-J / 2$, and the highest exited state corresponding to $\epsilon_{2^{N}-1}=J / 2$ energy (which is also nondegenerate) reads $\left|G_{+}\right\rangle$. Thus, the Hamiltonian (9) fulfills W-O TC definition [12] in the thermodynamic limit. It is worth noting that one could take a correlation function of the form $\left\langle E_{0}\left|\sigma_{z}^{(i)}(t) \sigma_{z}^{(j)}(0)\right| E_{0}\right\rangle$ and get absolutely the same result for the correlation function (1), namely, $e^{-i J t}$ (the two correlation functions coincide for an arbitrary $N$ ). Thus, the provided Hamiltonian (9) implements a genuine time crystal formally bypassing the W-O no-go theorem by exploiting nonlocality. We also note that long-range multispin interaction Hamiltonians may be potentially studied in the systems with slow but strong driving, where heating is precluded $[43,44]$.

Finally, we can ask the question: what is the most local Hamiltonian that satisfies TC conditions described after Eq. (2)? To answer, one needs to find the Hamiltonian contained in the spectrum $\left|G_{+}\right\rangle$and the shortest interaction range. The ultimate bound on such an interaction was given in Ref. [45], where the most local Hamiltonian comprises of at least $[N / 2]$-length strings. It can be written as $\hat{H}(J)=\hat{H}_{0}+J \hat{H}_{1}$, and contains the nearest-neighbor Ising Hamiltonian

$$
\hat{H}_{0}=-\sum_{j=1}^{N} \sigma_{z}^{(j)} \sigma_{z}^{(j+1)}
$$

for the periodic boundary conditions $\left(\sigma_{z}^{(N+1)} \equiv \sigma_{z}^{(1)}\right)$, and spin-string interaction part

$$
\hat{H}_{1}=\sigma_{x}^{(1)} \sigma_{x}^{(2)} \ldots \sigma_{x}^{([N / 2])}-\sigma_{x}^{([N / 2]+1)} \ldots \sigma_{x}^{(N)},
$$

which involves the half of the qubits. The ground state $|\mathrm{GS}\rangle$ of the Hamiltonian $\hat{H}(J)$ satisfies $M_{z}|\mathrm{GS}\rangle \sim\left|G_{+}\right\rangle$and $\left\langle\mathrm{GS}\left|M_{z}^{2}\right| \mathrm{GS}\right\rangle$ is finite at $N \rightarrow \infty$. These states have the 
energies $\hat{H}(J)\left|G_{+}\right\rangle=-N\left|G_{+}\right\rangle$and $\hat{H}(J)|\mathrm{GS}\rangle=[-N-$ $\left.2\left(\sqrt{1+J^{2}}-1\right)\right]|\mathrm{GS}\rangle$. The correlation function (1) for the Hamiltonian $\hat{H}(J)$ is given by

$$
\begin{aligned}
& \lim _{N \rightarrow \infty}\left\langle\mathrm{GS}\left|e^{i \hat{H}(J) t} \hat{M}_{z} e^{-i \hat{H}(J) t} \hat{M}_{z}\right| \mathrm{GS}\right\rangle \\
& =\lim _{N \rightarrow \infty}\left\langle\mathrm{GS}\left|M_{z}^{2}\right| \mathrm{GS}\right\rangle e^{-i 2\left(\sqrt{1+J^{2}}-1\right) t} \\
& =\sin ^{2}\left(\frac{3 \pi}{8}\right) e^{-i 2\left(\sqrt{1+J^{2}}-1\right) t}
\end{aligned}
$$

thus corresponding to the time crystalline behavior for the most local TC Hamiltonian $\hat{H}(J)$. In the absence of $\hat{H}_{1}$ the Hamiltonian $\hat{H}(J)$ reduces to the Ising Hamiltonian having $\left|G_{+,-}\right\rangle$as degenerate ground states.

Stability of the time crystal. - The important feature of the time crystal phase is its stability towards perturbations in the Hamiltonian (extra magnetic field), which in the case of DTC has allowed us to distinguish it from an otherwise trivial spin flip operation [14,16]. Examining the behavior of the Hamiltonian $\hat{H}(J)$ under magnetic field perturbations, $\hat{H}^{\prime}=\hat{H}(J)+\delta \hat{H}$ with $\delta \hat{H}=\sum_{j}\left(h_{x}^{j} \sigma_{x}^{(j)}+h_{y}^{j} \sigma_{y}^{(j)}+h_{z}^{j} \sigma_{z}^{(j)}\right)$, we note that $\langle\mathrm{GS}|\delta \hat{H}| \mathrm{GS}\rangle=0$ and $\left\langle G_{+}|\delta \hat{H}| G_{+}\right\rangle=0$. Moreover, the perturbation of the form $\delta \hat{H}=\sum_{j} \sigma_{x, y}^{(j)} \sigma_{x, y}^{(j+1)}$ analogously does not overlap with $|\mathrm{GS}\rangle$ or $\left|G_{+}\right\rangle$. Thus, these types of perturbations do not affect the part of the Hamiltonian that is responsible for TC behavior, (a)

(c)

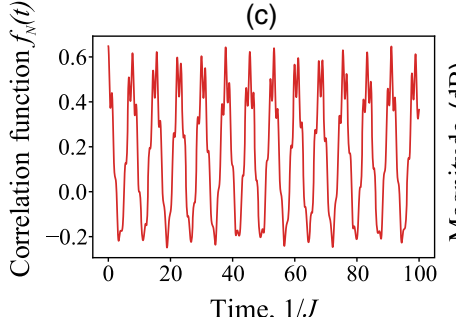

Time, $1 / J$

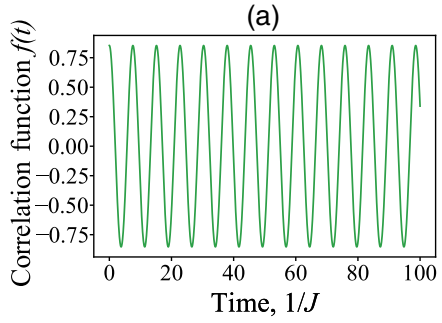

(b)

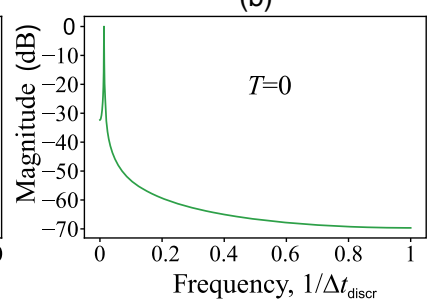

(d)

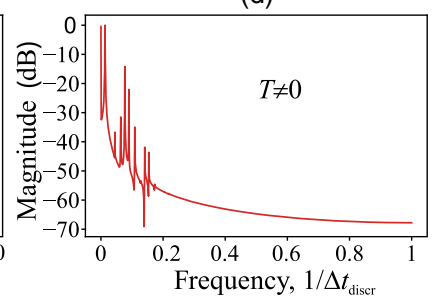

FIG. 1. Plot (a) shows the correlation function corresponding to TC (time crystal) behavior at zero temperature $T=0$ for an arbitrary size of the system $N$ and (b) shows its spectrum $\left(\Delta t_{\text {discr }}\right.$ is the discretization step in time). The deviation from the ideal TC behavior at $T \neq 0\left[\beta=(k T)^{-1}=1\right]$ is shown in the panel (c) for the finite system of $N=10$ sites, and its spectrum is in (d). In this case, the TC behavior is expected to vanish in the thermodynamic limit, when the number of additional harmonics tends to infinity, ruining the periodicity of the correlation function, whereas for the finite sized system the correlation function remains periodic with large but finite period.
$\hat{H}_{01}=\epsilon_{0}\left|E_{0}\right\rangle\left\langle E_{0}\left|+\epsilon_{1}\right| E_{1}\right\rangle\left\langle E_{1}\right|$ [for $\hat{H}(J)$ the ground state is $\left|E_{0}\right\rangle \equiv|\mathrm{GS}\rangle$ and $\left.\left|E_{1}\right\rangle \sim\left|G_{+}\right\rangle\right]$.

If the temperature is nonzero, then the analysis of stability becomes more subtle as the excited states come into play and contribute to the correlation function, leading to the generation of higher harmonics. Therefore, it is important to understand the scaling of the number of additional distinct harmonics with the size of the system. At nonzero temperature, our investigation of the Hamiltonian $\hat{H}(J)$ is limited to numerical analysis based on exact diagonalization which showed that the number of additional distinct harmonics grows with the size of the system. Thus, we expect the melting of the time crystal at nonzero temperature, which is shown in Fig. 1. However, the analysis of the TC phase at nonzero temperature in the thermodynamic limit remains an open question.

Relation to discrete time crystals.-To better understand the origin of TC behavior for continuous time, we can study its relation to the discrete counterpart [14]. We recall that standard DTC protocol is based on the unitary operation comprising a sequence of gates for an $N$-qubit chain. It reads as follows: (i) start with all-down or all-up spin string and apply spin flip operator $\hat{U}_{X}=\exp \left(-i \pi / 2 \sum_{j} \sigma_{x}^{(j)}\right)=$ $\prod_{j}\left(-i \sigma_{x}^{(j)}\right)$; (ii) evolve the state with the Ising Hamiltonian $\hat{U}_{\text {Ising }}=\exp \left(-i \tau \sum_{j=1}^{N} J_{j, j+1} \sigma_{z}^{(j)} \sigma_{z}^{(j+1)}\right)$, thus completing the single step unitary $\hat{\mathcal{U}}_{\text {step }}=\hat{U}_{\text {Ising }} \cdot \hat{U}_{X}$; (iii) continue for many stroboscopic periods $\hat{\mathcal{U}}_{\text {DTC }}=\hat{\mathcal{U}}_{\text {step }} \cdot \hat{\mathcal{U}}_{\text {step }} \cdot \ldots$. The algorithm is sketched in Fig. 2(a). The resulting stroboscopic dynamics then shows persistent oscillations of $M_{z}$ magnetization with period twice bigger than the drive $\prod_{j}\left(-i \sigma_{x}^{(j)}\right)$. Given the unitary operation, we can formally define the static Hamiltonian which governs the dynamics as (a)

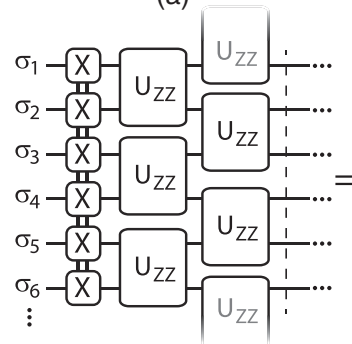

(b)

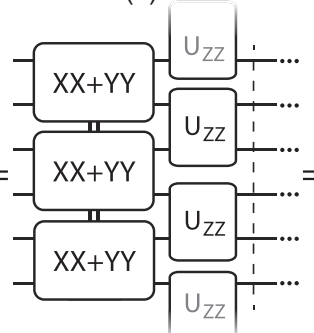

(c)

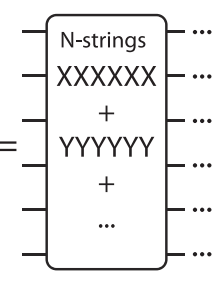

FIG. 2. The protocol for Floquet time crystal generation, and associated reasoning for existence of continuous time crystals with static $\hat{\mathcal{H}}_{\mathrm{TC}}$. The full digital sequence is presented in (a), which consists of $\pi$ rotations around the $x$ axis, corresponding to spin flip operators $(X)$ forming a string. The unitary corresponding to Ising interaction is divided into $U_{Z Z}$ commuting pieces. Next, the $X$ string and odd sublattice Ising terms are combined, leading to the product of $X X+Y Y:=-\cos \left(\phi_{j} / 2\right) \sigma_{x}^{(j)} \sigma_{x}^{(j+1)}-$ $\sin \left(\phi_{j} / 2\right) \sigma_{y}^{(j)} \sigma_{y}^{(j+1)}$ operators (b). Finally, during the last step all layers are linked together, forming the string (c). 
$\hat{H}_{\text {DTC }}=i \log \left(\hat{\mathcal{U}}_{\text {step }}\right)$. Generally, the logarithm can be computed using the Dynkin's formula, but the infinite order resummation needed to obtain the closed form expression seems infeasible. Instead, we can analyze the structure of $\hat{H}_{\text {DTC }}$ by studying the unitary operator in Fig. 2(a). First, exploiting Euler's formula we can combine the $\sigma_{x}$ string and the layer of Ising terms into a single block. Denoting phases as $J_{j, j+1} \tau=\phi_{j}$, the odd $j$ sublattice unitary reads $\prod_{j}(-1)\left\{\cos \left(\phi_{j} / 2\right) \sigma_{x}^{(j)} \sigma_{x}^{(j+1)}+\sin \left(\phi_{j} / 2\right) \sigma_{y}^{(j)} \sigma_{y}^{(j+1)}\right\}$, which we denote as blocks " $X X+Y Y$ " in Fig. 2(b). These blocks multiplied altogether contain various $\sigma_{x}$ and $\sigma_{y}$ strings, from length 2 to length $N$. Finally, the multiplication of the second sublattice Ising interaction either leaves string unaltered, or changes $\sigma_{x} \leftrightarrow \sigma_{y}$ (with some phase factor). Once the matrix $\hat{\mathcal{U}}_{\text {step }}$ is obtained, the effective Hamiltonian can be inferred employing the replica trick [46]. This relies on taking formally the limit

$$
\log \hat{\mathcal{U}}_{\text {step }}=\lim _{\rho \rightarrow 0} \frac{1}{\rho}\left(\hat{\mathcal{U}}_{\text {step }}^{\rho}-1\right)
$$

and corresponds to taking the powers of a previously derived unitary matrix. In this case, the odd powers contribute to the same stings we observe in the Hamiltonian (9) [see Fig. 2(c)], albeit with different prefactors, and cross terms from even powers contribute to the $\sigma_{z}$ strings of even length.

Finally, let us relate the physics of discrete time crystals through the properties of the $\hat{H}_{\mathrm{DTC}}$ Hamiltonian and continuous time crystal Hamiltonian described in the previous sections. In particular, if the ground state for both Hamiltonians is all-down or all-up spin string or their superposition, than one may expect that breaking of continuous TTS is inherently related to breaking of the discrete TTS for the corresponding Floquet TC. To yield direct comparison, we can consider the odd size $N$ system with Ising interaction fixed $\phi_{j, j+1}=J_{j, j+1} \tau=-1 / N$. Then, the matrix exponentials and logarithm can be calculated numerically, and their analysis provides a concise structure of the effective DTC Hamiltonian

$\hat{H}_{\mathrm{DTC}}=-\frac{1}{N} \sum_{j=1}^{N} \sigma_{z}^{(j)} \sigma_{z}^{(j+1)}+(-1)^{(N-1) / 2} \frac{\pi}{2} \prod_{j} \sigma_{x}^{(j)}$,

which is analogous to the time crystal Hamiltonian $\hat{H}(J)$ introduced earlier in a sense that it has a form of the Ising Hamiltonian plus a nonlocal part lifting the degeneracy of the ground state. For $N=4 k+3$ the nondegenerate ground state of the Hamiltonian (14) is $\left|G_{+}\right\rangle$and $\left|G_{-}\right\rangle$ is an exited state, whereas for $N=4 k+1$ it is the other way round. The energy difference between $\left|G_{ \pm}\right\rangle$states is equal to $\pi$ for any odd $N$. Therefore, the Hamiltonian (14) is nothing but a quantum time crystal and the corresponding correlation function for any odd $N$ is $f(t)=e^{-i \pi t}$.
Conclusions and outlook.-While previously it was believed to be impossible in the case of a nondriven system, we have shown that time crystals can emerge for Hamiltonians with long-range interactions in the form of spin strings. We described the minimal continuous TC Hamiltonian as a sum of strings encompassing halves of a spin system, and find that at zero temperature TC is stable. Finally, we note that nonlocal many-body operators that induce time crystalline behavior represent a specific example of Hermitian quantum behemoths [47], possibly relating the two notions.

The work was supported by the Government of the Russian Federation through the ITMO Fellowship and Professorship Program, Megagrant 14.Y26.31.0015, and Goszadanie No. 3.2614.2017/4.6 and 3.1365.2017/4.6 of the Ministry of Education and Science of Russian Federation. The authors acknowledge the help from the NORDITA Visiting Ph.D. program. We are grateful to Frank Wilczek and Anatoli Polkovnikov for useful discussions. We also would like to thank Ivan Iorsh for the fruitful suggestions.

[1] N. W. Ashcroft and N. David Mermin, Solid State Physics (Cengage Learning, Boston, MA, 1976), ISBN 9780030839931.

[2] P. Lodahl, S. Mahmoodian, and S. Stobbe, Interfacing single photons and single quantum dots with photonic nanostructures, Rev. Mod. Phys. 87, 347 (2015).

[3] E. Istrate and E. H. Sargent, Photonic crystal heterostructures and interfaces, Rev. Mod. Phys. 78, 455 (2006).

[4] I. Bloch, J. Dalibard, and W. Zwerger, Many-body physics with ultracold gases, Rev. Mod. Phys. 80, 885 (2008).

[5] I. S. Beloborodov, A. V. Lopatin, V. M. Vinokur, and K. B. Efetov, Granular electronic systems, Rev. Mod. Phys. 79, 469 (2007).

[6] A. Shapere and F. Wilczek, Classical Time Crystals, Phys. Rev. Lett. 109, 160402 (2012).

[7] F. Wilczek, Quantum Time Crystals, Phys. Rev. Lett. 109, 160401 (2012).

[8] F. Wilczek, Superfluidity and Space-Time Translation Symmetry Breaking, Phys. Rev. Lett. 111, 250402 (2013).

[9] T. Li, Z.-X. Gong, Z.-Q. Yin, H. T. Quan, X. Yin, P. Zhang, L.-M. Duan, and X. Zhang, Space-Time Crystals of Trapped Ions, Phys. Rev. Lett. 109, 163001 (2012).

[10] P. Bruno, Comment on "Quantum Time Crystals": A New Paradigm or Just Another Proposal of Perpetuum Mobile?, Phys. Rev. Lett. 110, 118901 (2013).

[11] P. Bruno, Impossibility of Spontaneously Rotating Time Crystals: A No-Go Theorem, Phys. Rev. Lett. 111, 070402 (2013).

[12] H. Watanabe and M. Oshikawa, Absence of Quantum Time Crystals, Phys. Rev. Lett. 114, 251603 (2015).

[13] K. Sacha, Modeling spontaneous breaking of timetranslation symmetry, Phys. Rev. A 91, 033617 (2015).

[14] D. V. Else, B. Bauer, and C. Nayak, Floquet Time Crystals, Phys. Rev. Lett. 117, 090402 (2016). 
[15] V. Khemani, A. Lazarides, R. Moessner, and S. L. Sondhi, Phase Structure of Driven Quantum Systems, Phys. Rev. Lett. 116, 250401 (2016).

[16] N. Y. Yao, A. C. Potter, I.-D. Potirniche, and A. Vishwanath, Discrete Time Crystals: Rigidity, Criticality, and Realizations, Phys. Rev. Lett. 118, 030401 (2017).

[17] D. V. Else, B. Bauer, and C. Nayak, Prethermal Phases of Matter Protected by Time-Translation Symmetry, Phys. Rev. X 7, 011026 (2017).

[18] B. Huang, Y.-H. Wu, and W. V. Liu, Clean Floquet Time Crystals: Models and Realizations in Cold Atoms, Phys. Rev. Lett. 120, 110603 (2018).

[19] W. Chi Yu, J. Tangpanitanon, A. W. Glaetzle, D. Jaksch, and D. G. Angelakis, Discrete time crystal in globally driven interacting quantum systems without disorder, Phys. Rev. A 99, 033618 (2019).

[20] J. Zhang, P. W. Hess, A. Kyprianidis, P. Becker, A. Lee, J. Smith, G. Pagano, I.-D. Potirniche, A. C. Potter, A. Vishwanath, N. Y. Yao, and C. Monroe, Observation of a discrete time crystal, Nature (London) 543, 217 (2017).

[21] S. Choi, J. Choi, R. Landig, G. Kucsko, H. Zhou, J. Isoya, F. Jelezko, S. Onoda, H. Sumiya, V. Khemani, C. von Keyserlingk, N. Y. Yao, E. Demler, and M. D. Lukin, Observation of discrete time-crystalline order in a disordered dipolar many-body system, Nature (London) 543, 221 (2017).

[22] S. Autti, V. B. Eltsov, and G. E. Volovik, Observation of a Time Quasicrystal and Its Transition to a Superfluid Time Crystal, Phys. Rev. Lett. 120, 215301 (2018).

[23] S. Pal, N. Nishad, T. S. Mahesh, and G. J. Sreejith, Temporal Order in Periodically Driven Spins in Star-Shaped Clusters, Phys. Rev. Lett. 120, 180602 (2018).

[24] J. Rovny, R. L. Blum, and S. E. Barrett, Observation of Discrete-Time-Crystal Signatures in an Ordered Dipolar Many-Body System, Phys. Rev. Lett. 120, 180603 (2018).

[25] R. W. Bomantara and J. Gong, Simulation of Non-Abelian Braiding in Majorana Time Crystals, Phys. Rev. Lett. 120, 230405 (2018).

[26] J. O'Sullivan, O. Lunt, C. W. Zollitsch, M. L. W. Thewalt, J. J. L. Morton, and A. Pal, Dissipative discrete time crystals, arXiv:1807.09884.

[27] K. Giergiel, A. Dauphin, M. Lewenstein, J. Zakrzewski, and K. Sacha, Topological time crystals, New J. Phys. 21, 052003 (2019).

[28] K. Giergiel, A. Kuroś, and K. Sacha, Discrete time quasicrystals, Phys. Rev. B 99, 220303(R) (2019).

[29] P. Das, S. Pan, S. Ghosh, and P. Pal, Cosmological time crystal: Cyclic universe with a small cosmological constant in a toy model approach, Phys. Rev. D 98, 024004 (2018).

[30] P. Das, S. Pan, and S. Ghosh, Thermodynamics and phase transition in ShapereWilczek fgh model: Cosmological time crystal in quadratic gravity, Phys. Lett. B 791, 66 (2019).

[31] A. Kosior and K. Sacha, Dynamical quantum phase transitions in discrete time crystals, Phys. Rev. A 97, 053621 (2018).

[32] K. Sacha and J. Zakrzewski, Time crystals: A review, Rep. Prog. Phys. 81, 016401 (2018).

[33] D. V. Else, C. Monroe, C. Nayak, and N. Y. Yao, Discrete Time Crystals, arXiv:1905.13232.

[34] C. J. Turner, A. A. Michailidis, D. A. Abanin, M. Serbyn, and Z. Papić, Weak ergodicity breaking from quantum many-body scars, Nat. Phys. 14, 745 (2018).

[35] W. W. Ho, S. Choi, H. Pichler, and M. D. Lukin, Periodic Orbits, Entanglement, and Quantum Many-Body Scars in Constrained Models: Matrix Product State Approach, Phys. Rev. Lett. 122, 040603 (2019).

[36] S. Choi, C. J. Turner, H. Pichler, W. W. Ho, A. A. Michailidis, Z. Papić, M. Serbyn, M. D. Lukin, and D. A. Abanin, Emergent SU(2) Dynamics and Perfect Quantum Many-Body Scars, Phys. Rev. Lett. 122, 220603 (2019).

[37] T. Iadecola, M. Schecter, and S. Xu, Quantum many-body scars and space-time crystalline order from magnon condensation, arXiv:1903.10517.

[38] M. Medenjak, B. Buca, and D. Jaksch, The isolated Heisenberg magnet as a quantum time crystal, arXiv: 1905.08266.

[39] B. Buča, J. Tindall, and D. Jaksch, Non-stationary coherent quantum many-body dynamics through dissipation, Nat. Commun. 10, 1730 (2019).

[40] F. M. Gambetta, F. Carollo, M. Marcuzzi, J. P. Garrahan, and I. Lesanovsky, Discrete Time Crystals in the Absence of Manifest Symmetries or Disorder in Open Quantum Systems, Phys. Rev. Lett. 122, 015701 (2019).

[41] G. Tóth and O. Gühne, Entanglement detection in the stabilizer formalism, Phys. Rev. A 72, 022340 (2005).

[42] The origin lies in a non-trivial operator representation of many-body projectors, which can be checked by direct decomposition.

[43] A. Haldar, R. Moessner, and A. Das, Onset of Floquet thermalization, Phys. Rev. B 97, 245122 (2018).

[44] A. Lazarides, A. Das, and R. Moessner, Equilibrium states of generic quantum systems subject to periodic driving, Phys. Rev. E 90, 012110 (2014).

[45] P. Facchi, G. Florio, S. Pascazio, and F. V. Pepe, Greenberger-Horne-Zeilinger States and Few-Body Hamiltonians, Phys. Rev. Lett. 107, 260502 (2011).

[46] S. Vajna, K. Klobas, T. Prosen, and A. Polkovnikov, Replica Resummation of the Baker-Campbell-Hausdorff Series, Phys. Rev. Lett. 120, 200607 (2018).

[47] I. M. Khaymovich, M. Haque, and P. A. McClarty, Eigenstate Thermalization, Random Matrix Theory, and Behemoths, Phys. Rev. Lett. 122, 070601 (2019). 